\title{
Carga laboral y el Síndrome Burnout en el personal administrativo y docente universitario
}

\author{
Workload and Burnout Syndrome in university administrative and teaching staff
}

Carga de trabalho e Síndrome de Burnout em pessoal administrativo e docente universitário

Marisol Nancy Rodríguez Villanueva

marsol_2906@hotmail.com

https://orcid.org/0000-0003-3854-3679

Universidad Peruana Unión, Lima, Perú
Guido Angelo Huapaya Flores

angelo.huapaya@upeu.edu.pe

https://orcid.org/0000-0002-1237-2886

Universidad Peruana Unión, Lima, Perú

Recibido: octubre 2020 / Revisado: noviembre 2020 / Aceptado: diciembre 2020 / Publicado: enero 2021

RESUMEN

El objetivo del estudio fue determinar la diferencias en la relación de la carga laboral y los rasgos del síndrome de Burnout en el personal docente y administrativo de la Universidad Peruana Unión, Lima, 2017. Los resultados evidencia también que no existe diferencias del Burnout entre el personal contratado y nombrado; es decir que tanto en el personal contratado y nombrado existe la misma exposición a rasgos de burnout como de carga laboral. Se halló diferencias significativas es decir tanto los que vienen laborando entre 1 a 5 años como los que tienen más de 6 años no están expuestos al mismo nivel de síndrome de Burnout. En conclusión se visualiza que la carga laboral está incidiendo en que los trabajadores presenten rasgos de burnout aunque no están en niveles altos están en grados casi riesgosos aspecto que podría repercutir en un futuro en problemas de salud como ya se evidencia en las tablas descriptivas.

Palabras clave:

Burnout; carga laboral; estrés; agotamiento emocional
The objective of the study was to determine the differences in the relationship between workload and the traits of Burnout syndrome in the teaching and administrative staff of the Universidad Peruana Unión, Lima, 2017. The results also show that there is no difference in Burnout between hired and appointed personnel; that is to say that in both hired and appointed personnel there is the same exposure to burnout and workload traits. Significant differences were found, i.e. both those who have been working for 1 to 5 years and those who have been working for more than 6 years are not exposed to the same level of Burnout syndrome. In conclusion, it can be seen that the workload is influencing the workers to present burnout traits, although they are not at high levels, they are at almost risky levels, an aspect that could have repercussions in the future in health problems, as is already evident in the descriptive tables.

Key words:

Burnout; workload; stress; emotional exhaustion
O objetivo do estudo foi determinar as diferenças na relação da carga de trabalho e as características da Síndrome de Burnout no corpo docente e administrativo da Universidad Peruana Unión, Lima, 2017. Os resultados também mostram que não há diferenças no Burnout entre o pessoal contratado e o nomeado; Ou seja, tanto no pessoal contratado quanto no nomeado há a mesma exposição aos traços de burnout e carga de trabalho. Foram encontradas diferenças significativas, ou seja, tanto aqueles que trabalham entre 1 a 5 anos quanto aqueles que têm mais de 6 anos não estão expostos ao mesmo nível de síndrome de Burnout. Em conclusão, visualizase que a carga de trabalho está influenciando os trabalhadores a apresentarem traços de burnout, embora não se encontrem em níveis elevados, encontram-se em graus quase de risco, aspecto que pode ter repercussões futuras em agravos à saúde como já é evidente em as tabelas descritivas.

Palavras-chave:

Burnout; carga de trabalho; estresse; Exaustão emocional 


\section{INTRODUCCIÓN}

La Organización Panamericana de la Salud, Organización Mundial de la Salud (OPS y OMS), la Organización Internacional del Trabajo (OIT) y la Organización de Estados Americanos llevaron a cabo un seminario especial el 28 de abril para memorar el Día Mundial de la Seguridad y la Salud en el Trabajo, sobre el impacto del Bournout que es estrés laboral en la salud del trabajador y para minorizar el problema.. La Dra. Rodríguez, asesora Regional en Salud de los Trabajadores de la OPS y OMS, aludió que estos problemas son capaces de reducir la motivación, el compromiso y el rendimiento laboral; y generan un aumento del ausentismo, la rotación de personal y el retiro temprano.

La Dr. Fortune, Jefe Interino del Programa Especial en Desarrollo Sostenible y Equidad en Salud de la OPS y OMS refirió que estudio de las condiciones de trabajo, convergen un conjunto de dimensiones sociales, personales y físicos donde trabajan los docentes, la salud es un concepto complejo y un equilibrio social, psicológico, fisiológico y biológico que influye en los docentes que acuden a laborar.

Unión Nacional de Educadores (UNE, 2005) refiere que en este "estudio de investigación de las condiciones de trabajo, convergen un conjunto de dimensiones sociales, personales y físicos donde laboran los docentes, la salud es un concepto integral y un equilibrio social, psicológico, fisiológico y biológico que influye, fuertemente en los docentes acuden a trabajar." Algunos estudios realizados en Latinoamérica y otros realizados en Argentina, Chile, Ecuador, México, representan una voz de alarma en el sistema educativo y la sociedad que ofrecen hallazgos múltiples, en particular relacionados con la enfermedades de la salud mental como síndrome de burnout, depresión, neurosis y una diversidad de enfermedades psicosomáticas diagnosticadas y percibidas (gastritis, úlceras, colon irritable, entre otras).
Ministerio de salud (2004) actualmente, la salud no se entiende como la ausencia de enfermedades solamente. La organización mundial de la salud, define que la salud mental como el estado de bienestar que permite a cada individuo realizar su potencial, enfrentarse a las dificultades usuales de la vida, trabajar productiva y fructíferamente y contribuir con su comunidad. Los resultados que no son abordadas contribuirán a reproducir de generación en generación este problema. El estudio epidemiológico Metropolitano de Salud Mental.

Mansilla, s.a los trabajadores que frecuentemente realizan ocupaciones en una atención directa y ayuda a personas constante son más susceptibles al síndrome de burnout; como: "los profesionales de salud, sanitarias, sociales o educativas, y son algunos de estos trabajadores los que pueden desarrollar sentimientos cada vez más negativos hacia los pacientes, usuarios o alumnos". El síndrome se ha considerado tan prioritario en servicios sanitarios, sociales, instituciones universitarias en docentes, administrativos y servicios de seguridad (Elloy, Terpening y Kohls, 2001).

El Instituto de Estudios Laborales (IEL) "ha realizado una investigación en Europa en el que se reseña que el $87,8 \%$ de los médicos de Atención Primaria se siente agotado emocionalmente" (Diario Médico, 2004).

Barraza (2013) refiere que sus estudios de investigaciones fueron realizadas en México en la Red Nacional e Internacional de factores psicosociales. Igualmente expresó que abordan factorespsicosocialeseneltrabajo,ycomoresultado determinan que los docentes de la Universidad Automa de Nayarit (UAN) se ven afectados en su salud mental por la carga de trabajo. Es el resultados de la excesiva carga de trabajo que se relaciona con el síndrome de burnout y cuestiones de redes sociales, que se han sentido presionados por el cambio de rol que ha estado vigente, a partir de la reforma universitaria ya obtienen impacto 
en su salud mental y señaló que, esta investigación fue considerada el área Económico Administrativa y de la Salud, es un proyecto con algunas pautas, que institucionalmente se asumieron para mitigar los efectos que con lleva el exceso de trabajo, el cambio de rol en el docente y tiene como finalidad, tratar de evitar mayores daños a la salud."

\section{Referentes}

Vargas y Poveda (2017) realizaron un estudio de investigación y su objetivo fue "investigar la episodio del Síndrome de Burnout en el ejercicio laboral del personal administrativo de la Facultad de Ciencias Humanas y de la Educación de la Universidad Técnica de Ambato de la ciudad de Ambato provincia de Tungurahua y la metodología que usaron fue la aplicación de encuesta y test, tiene un enfoque cualitativo, cuantitativo, de modalidad bibliográfica, documental y de campo, resalta en los niveles el descriptivo y los resultados determinó que los profesionales presentan signos y síntomas relacionados con el Burnout, teniendo en cuenta que es una enfermedad silenciosa y que evoluciona constantemente; produce debilidades tanto físicas y psicológicas en la persona, impulsa conflictos con los usuarios que se relaciona durante las horas de labor y desarrolla problemas familiares". En conclusión se determinó que hay una relación directa en el Burnout y el desempeño laboral del personal administrativo, y si no recibe tratamiento le producirá signos y síntomas como: cansancio emocional, despersonalización y falta de realización personal, por tanto el investigador sitúa a consideración varias recomendaciones con el interés de favorecer no solo al personal administrativo, sino a la población de profesionales de similares características y usuarios en general de la Facultad de Ciencias Humanas y de la Educación de la Universidad Técnica de Ambato.

Marenco y Hernando (2016) realizaron un estudio de investigación, y su objetivo fue determinar la asociación entre las dimensiones de apoyo social con las manifestaciones del síndrome de burnout, en docentes de universidad del Caribe Colombiano y la metodología que se usó en esta investigación de diseño ex post facto participaron 235 docentes de diferentes instituciones educativas, la muestra fue evaluado mediante el instrumento Maslach Burnout Inventory-Educator Survery y el cuestionario MOS de apoyo social y los resultados que se hallaron fue que el $23 \%$ de los docentes aquejó agotamiento emocional y el $22.5 \%$ despersonalización. Los niveles de apoyo social se manifestaron con intensidad alta o media. Asimismo, se observaron asociaciones significativas entre las dimensiones de apoyo y la despersonalización. En conclusión se discute la discrepancia del apoyo social como un elemento relevante a considerar en la intervención preventiva del burnout en los profesores.

\section{Marco teórico}

Monte, (2008) describe que el Síndrome de Burnout, es un resultado prolongado al estrés constante de sobrecarga laboral. En la Clasificación Internacional de las Padecimientos, decima traducción (CIE 10), el Síndrome corresponde al código de $Z$ 73.0, el cual presenta los siguientes síntomas:

Conductual: el comportamiento es suspicaz y paranoico, inflexible y rígido, con una incapacidad para estar relajado.

- Somáticos: fatiga crónica, resaltan los frecuentes dolores de cabeza, espalda, cuello y musculares, insomnio, alteraciones respiratorias, gastrointestinales e hipertensión.

- Emocionales: se expresa con hostilidad, irritabilidad y odio, dificultad para controlar y demostrar emociones, aburrimiento, impaciencia y depresión. 
Cognitivo: presenta baja autoestima, disminución de la realización personal en el trabajo, fracaso profesional e impotencia para el progreso profesional, (citado por Muñoz, Osorio, Robles, Romero, 2014).

Según Maslach en 1982, puntualiza que el síndrome de Burnout es "determinado por agotamiento emocional, despersonalización y baja realización personal". El individuo que realiza trabajos con el servicio público como docente, administrativo y sanitario.

Las características del Síndrome de Burnout para Maslach concretan como:

- Cansancio emocional (CE): es caracterizado por la disminución de energía, desgaste de los recursos emocionales, agotamiento y fatiga con expresiones como físicas y psíquicas debido al trabajo que el sujeto realiza.

- Despersonalización (DP): presenta y desarrollo actitudes negativas, dureza con los demás (deshumanización), con lleva a irritación y deterioro de motivación.

- Disminución de la realización personal (RP): "presenta una actitud negativa hacia su persona y disminuye la capacidad de labor con la caída de la productividad, imposibilidad para resistir el estrés ambiental" (citado por Rodríguez, et al., 2008).

\section{Sintomatología del Síndrome de Burnout}

Las dimensiones son importantes del Síndrome de Burnout por que se relacionan con los efectos fisiológicos, psicológicos y conductuales que dicho síndrome induce en el profesional docentes, administrativos y salud (citado por Rodríguez, 2009).

\section{Síntomas relacionales}

Se observa aislamiento del personal docente
y administrativo, así como, irritabilidad al relacionarse con otros (Arón y Llanos, 2004) como también confrontaciones reiteradas con colegas, y maltrato al que trabaja con quien padece Burnout, en el caso de docentes y administrativo (Catsicaris et al., 2007). En general, existe un retraimiento de las demandas y las relaciones, donde se dan actitudes de desprecio hacia uno mismo y hacia "el mundo" (Längle, 2010).

\section{Síntomas organizacionales}

Más allá del mismo sujeto, secciones completas de trabajadores pueden manifestar la presencia del síndrome mediante un aumento de la sobre carga de trabajo, competencia entre grupos, poca colaboración y la demostración de rabia o impaciencia entre colegas (Aguirre y Montedónico, 2009).

\section{Consecuencias del Síndrome de Burnout}

López y Bernal (2002) y Legorreta (2004) refieren que todos las cambios trascienden en el desempeño profesional, en el trabajo que brinda ella personal docente y administrativo dentro de la misma institución se observa, aumento del ausentismo, apatía hacia la organización, aislamiento, disminución de la eficacia del trabajo, actitud cínica y fatiga emocional, aparición de situaciones depresivas que aumentan la utilización de líquidos excitantes como: café, alcohol, barbitúricos y comidas, cigarrillo y por último comprometen el ambiente familiar. Como se puede apreciar es común que el síndrome de Burnout se presenta en profesionales docentes, administrativos y salud, poniendo en mayor riesgo los alumnos y el ambiente laboral, que es producido por la demasiada carga de trabajo, la falta de motivación y de reconocimiento produce 
resultados desconformidad. Otros trabajos de investigación sustentan que va enfocado a identificar la prevalencia del síndrome de Burnout y conocer cuáles son las causas que lo producen dentro de las Instituciones Públicas de Salud en México (citado por Rodríguez, et al., 2009).

\section{Diagnóstico}

El Manual Diagnóstico y Estadístico de los Trastornos Mentales (DSM IV, 2002) lo clasifica el síndrome de burnout según la categoría de los Trastornos Adaptativos. Estos Trastornos tienen característica principal el progreso de sintomatologías emocionales o comportamiento de respuestas en un estresante psicosocial identificable. Debe ser resuelto dentro los siguientes seis meses que siguen a su desaparición del estresante o sus consecuencias, sin embargo esto puede variar si el factor estresante es agudo o crónico y dentro de los subtipos que se encuentran los rasgos ansiosos, depresivos y mixtos, este síndrome, que corresponde a reacciones desadaptadas a estresantes psicosociales, de curso crónico, asimismo la Organización Mundial de la Salud a clasificado este síndrome dentro del capítulo $V$ del Manual, específicamente en el apartado XXI referente a "factores que influyen en el estado de salud y en el contacto con los servicios de salud" y en otro apartado como "problemas relacionados con el manejo de las dificultades de la vida", comprensible como agotamiento (código Z73.0) (Bosqued, 2008).

\section{Carga laboral}

Maslach y Leiter (2005) explican que una alta demanda laboral en un solo individuo genera una gran sobrecarga, pudiendo desgastar gran parte de su energía resultando imposible su recuperación. Esta complicación en la carga laboral puede presentarse también en casos en que se ha escogido erróneamente el tipo de trabajo pudiendo ser especialmente complejo aquellos 26 casos donde el trabajo del profesional consiste en expresar y demostrar emociones inconsistentes con las suyas. Generalmente la carga laboral va asociada al aspecto de desgaste emocional del Burnout (Citado por Wenner, 2010).

\section{Comunidad}

El conflicto se da en este caso, cuando la persona no puede establecer una conexión positiva con otros en el lugar de trabajo, donde hay conflictos que se dan de manera crónica y nunca son resueltos, existiendo un ambiente de hostilidad y frustración reduciendo la posibilidad de tener algún apoyo social (Citado por Wenner, 2010).

\section{Equidad}

Maslach y Leiter (2005). Expresan que el respeto mutuo entre los profesionales es central para el compartido sentido de comunidad. La inequidad se puede dar de muchas maneras, a través de un salario injusto, cuando hay engaño, cuando las evaluaciones y los ascensos son manejados de manera poco apropiada. Una carencia de equidad posibilita el desarrollo del Burnout en por lo menos dos días; una de ellas es la experiencia de un tratamiento desigual que resulta emocionalmente desgastante y segunda la inequidad estimula un comportamiento despersonalizado en el lugar de trabajo (Citado por Wenner, 2010).

\section{Valores}

Siguiendo con Maslach y Leiter (2005), el sujeto puede encontrarse realizando trabajos que son poco éticos para ellos y poco acordes a sus propios valores. También puede darse un desajuste entre las propias expectativas y aspiraciones del profesional y los valores de la organización. Los profesionales también pueden encontrarse en un conflicto cuando existe una fuerte discrepancia entre lo que dice el estamento de la organización 
y lo que ocurre en la práctica laboral misma, o bien cuando los valores dentro de la organización misma se contradicen (Citado por Wenner, 2010).

\section{Factores intervinientes en el Burnout Laboral}

- Recursos personales: el trabajador presentan cualidades como "habilidades, anhelos, insuficiencias, valores, autoeficacia, autocontrol.

- Recursos laborales: disminuye los costos, las demandas y estimulan el incremento trabajador, enseñanza y logro de metas.

- Demandas laborales: entendidas así el trabajo como "un esfuerzo físico, mental y emocional y por tanto posee costos físicos y psicológicos" (Citado por Sánchez, 2011).

Características del trabajo

Características del puesto

- Tareas monótonas, tediosas y tribales.

. Falta de variedad.

- Tareas desagradables.

- Tareas que causan antipatía.

Volumen y ritmo del trabajo

- Desproporción de trabajo.

. Trabajos con términos muy exactos.

Horarios de trabajo

. Horarios de trabajo preciso e intolerante.

. Jornadas de labor excesiva u horario extras.

. Horarios de trabajo casualidades.

. Métodos de turnos mal ubicados.

Participación en la toma de decisiones

. Disminución de intervención en la toma de decisiones.

. Deterioro de interposición en el ritmo laboral, el horario y el entorno laboral.
Perspectivas profesionales, estatus y salario

. Incertidumbre laboral.

- Alta de perspectivas de promoción profesional.

. Promoción excesiva o insuficiente.

- Actividad disminuida en la valoración social.

. Remuneración por trabajo o destajo.

. Sistema de evaluación, ventajas injustas o insuficientes.

- Demasía o escasez de capacidades para el puesto.

Papel en la entidad

. Documento indeterminado.

. Funciones confrontadas en el mismo cargo.

- Responsabilidad de recursos humanos.

- Atender asiduamente a otros individuos y resolver sus problemas.

Relaciones interpersonales

. Inspección impropia, incorrecta que no facilita apoyo.

- Deficiencia de relaciones interpersonales con los colegas.

. Intimidación, persecución y violencia.

- Trabajo aislado o insociable.

- Abandono de programaciones determinado para solucionar inconvenientes.

Cultura institucional

. Insuficiencia en la comunicación.

. Liderazgo incorrecto.

. Objetivos incorrectos en la organización.

Relación en la vida familiar y la vida laboral

. Exigencias confrontadas en la vida laboral y vida familiar.

- Déficit en el apoyo familiar en las dificultades del trabajo, (Citado por Sánchez, 2011).

Contexto laboral: 


\section{MÉTODO}

El presente estudio de investigación es de tipo observacional, no experimental porque no se manipuló ninguna variable. Su diseño fue comparativo porque se determinaron las diferencias significativas entre las variables; igualmente, asociativo y correlacional en vista que se analizó el grado de relación de las variables en la docentes y administradores; fue corte transversal por que la información fue recogida en un solo momento especifico.

\section{Población y muestra}

la población del estudio estuvo constituida por 560 personas, entre el personal docente y administrativo constituido por 560, entre el personal docente y administrativo en la universidad Peruana Unión. Por motivo circunstancial del contexto del desarrollo de actividades académicas y administrativas la muestra fue seleccionada de forma no aleatoria y no probabilística de acuerdo al criterio del investigador considerando los lineamientos de inclusión y exclusión: quedando la muestra en 229.

\section{Validación del instrumento original de carga laboral}

Ceballos, et al.., (2014) refieren que la validez de contenido de ESCAM fue a través de expertos del área de la psicología laboral, ergonomía y enfermería laboral, y una prueba piloto. Para estimar la validez de constructo, se realizó un análisis factorial exploratorio de componente principales con rotación ortogonal (Varimax). Se evaluó la validez de criterio concurrente, por medio de análisis de correlación, entre las dimensiones de ESCAM y SUSESO ISTAS-21 (Dimensión exigencias psicológicas). Indica la organización, el procesamiento y análisis estadístico de los datos se realizó con el software SPSS versión 15.0 para Windows XP.

\section{Instrumento Burnout}

El segundo" instrumento que se usó tuvo correspondencia a un cuestionario a un cuestionario, la escala de Maslach Bornout Invetory (MBI) instrumento se usó para medir estrés laboral crónico o Bornout. Constituido por 22 ítems en forma de afirmaciones sobre los sentimientos y actitudes del profesional en su trabajo y hacia los pacientes. Se divide en 3 dimensiones que es": a) Cansancio emocional (CE). "Constituye de 9 ítems que evalúa la vivencia de estar exhausto emocionalmente por las demandas del trabajo". b) Despersonalización (DP). Formada por 5 ítems. "Valora el grado en que cada uno reconoce actitudes de frialdad y distanciamiento hacia el paciente". c) Realización personal (RP). Compuesta por 8 ítems. "Evalúa los sentimientos de autoeficacia y realización personal en el trabajo". "Si la puntuación es alta, concretamente más de 31 puntos para CE y más de 13 para DP, indica un mayor grado de Burnout. En cambio, la escala de RP refleja mayores índices de Burnout cuando las puntuaciones son menores de 30 puntos". (Citado por Rodríguez, Fernández, Benítez, Camino y Brea, 2008).

Según Riveri, Sánchez, et al., (2008) "realizaron la medición de Burnout es el cuestionario de Maslach es una prueba específica para medir el desgaste, agotamiento e insatisfacción en el trabajo. Para obtener la puntuación" (s.n.) en:

- AE: Agotamiento emocional, se suman los números que ha valorado las preguntas $1,2,3$, $4,6,8,1314,16,20$.

- DP: Despersonalización, se continúa de igual con las preguntas $5,10,11,15,22$.

- LP: Logros personales, se realiza lo mismo con las preguntas $7,9,12,17,18,19,21$.

Para conocer el grado de estrés laboral de cada profesional docente se llevó a cabo la siguiente escala de medición de Burnout. 


\section{RESULTADOS}

En la Tabla 1 se observa en cuanto a los rasgos de posibilidad de presentar el Síndrome de Burnout el $41,5 \%$ no presenta ningún rasgo; el $42,4 \%$ presenta bajo rasgos de Burnout; el 14,8\% presenta rasgos medianamente; y sólo $1,3 \%$ presenta rasgos moderadamente altos.

\section{Resultados 1}

Tabla 1. Rasgos de posibilidad de presentar el Síndrome de Burnout.

\begin{tabular}{lcccc}
\hline & Frecuencia & Porcentaje & Porcentaje válido & $\begin{array}{c}\text { Porcentaje } \\
\text { acumulado }\end{array}$ \\
\hline No presenta ningún rasgos & 95 & 41,5 & 41,5 & 41,5 \\
Presenta bajo rasgos & 97 & 42,4 & 42,4 & 83,8 \\
presenta rasgos medianamente & 34 & 14,8 & 14,8 & 98,7 \\
Presenta suficientes rasgos & 3 & 1,3 & 1,3 & 100,0 \\
Total & $\mathbf{2 2 9}$ & $\mathbf{1 0 0 , 0}$ & $\mathbf{1 0 0 , 0}$ & \\
\hline
\end{tabular}

\section{Resultados 2}

En la tabla 2, se observa que la carga laboral en el personal de la UPeU presenta que el $67,7 \%$ tiene carga laboral suficiente; a diferencia del $29,7 \%$ que presenta tener carga laboral baja y sólo un 2,7\% indica tener alta carga laboral.

Tabla 2. Carga laboral en el personal de la UPeU.

\begin{tabular}{lcccc}
\hline & Frecuencia & Porcentaje & Porcentaje válido & $\begin{array}{c}\text { Porcentaje } \\
\text { acumulado }\end{array}$ \\
\hline Baja carga laboral & 68 & 29,7 & 29,7 & 29,7 \\
Suficiente carga laboral & 155 & 67,7 & 67,7 & 97,4 \\
Alta carga laboral & 6 & 2,6 & 2,6 & 100,0 \\
Total & $\mathbf{2 2 9}$ & $\mathbf{1 0 0 , 0}$ & $\mathbf{1 0 0 , 0}$ & \\
\hline
\end{tabular}

\section{Resultado 3 y 4}

Para que se presente síndrome de burnout tiene que darse dos condiciones: la dimensiones agotamiento emocional y despersonalización deberían estar en niveles elevados y la dimensión logros personales deberían presentarse en niveles bajos; sin embargo en las tablas se observa lo siguiente:
En la tabla 3, se describe que un poco más de la mitad de encuestados (166 individuos) presentan agotamiento emocional en niveles de regulares hacia abajo y los logros personales también están bajos. Lo que la teoría requiere que el agotamiento emocional se presente en niveles altos y logros bajos. Condición que no se cumple. 
En la tabla 4, se describe que casi la mitad de encuestados (91 individuos) igualmente presentan despersonalización en niveles de bajo y una buena proporción (47 individuos) no presentan despersonalización y sólo tan sólo (62 individuos) presentan niveles regulares y casi muy pocos 9 personas en niveles altos en relación a los logros personales que también se presentan de niveles regulares hacia abajo. Lo que la teoría requiere que la despersonalización se presente en niveles altos y logros bajos. Condición que no se cumple.

Por lo tanto el personal encuestados docentes y administrativos no presentan Síndrome de Burnout condición que es muy significativa $p$ valor 0,000 para ambas relaciones.

Tabla 3. Tabla de contingencia entre agotamiento emocional y logros personales para observar si se presenta el síndrome de burnout.

\begin{tabular}{|c|c|c|c|c|c|c|c|c|}
\hline & & & \multicolumn{5}{|c|}{ Logros personales } & \multirow[b]{2}{*}{ Total } \\
\hline & & & $\begin{array}{l}\text { No } \\
\text { presenta }\end{array}$ & Bajo & Regulares & Suficiente & Alto & \\
\hline \multirow{10}{*}{$\begin{array}{l}\text { Agotamiento } \\
\text { emocional }\end{array}$} & Bajo & Recuento & 25 & 23 & 4 & 1 & 2 & 55 \\
\hline & & $\begin{array}{l}\% \text { dentro de } \\
\text { agotamiento emocional }\end{array}$ & $45,5 \%$ & $41,8 \%$ & $7,3 \%$ & $1,8 \%$ & $3,6 \%$ & $100,0 \%$ \\
\hline & Regulares & Recuento & 16 & 52 & 31 & 7 & 5 & 111 \\
\hline & & $\begin{array}{l}\text { \% dentro de } \\
\text { agotamiento emocional }\end{array}$ & $14,4 \%$ & $46,8 \%$ & $27,9 \%$ & $6,3 \%$ & $4,5 \%$ & $100,0 \%$ \\
\hline & Suficiente & Recuento & 4 & 17 & 21 & 10 & 2 & 54 \\
\hline & & $\begin{array}{l}\text { \% dentro de } \\
\text { agotamiento emocional }\end{array}$ & $7,4 \%$ & $31,5 \%$ & $38,9 \%$ & $18,5 \%$ & $3,7 \%$ & $100,0 \%$ \\
\hline & Alto & Recuento & 1 & 4 & 3 & 0 & 0 & 8 \\
\hline & & $\begin{array}{l}\% \text { dentro de } \\
\text { agotamiento emocional }\end{array}$ & $12,5 \%$ & $50,0 \%$ & $37,5 \%$ & $0,0 \%$ & $0,0 \%$ & $100,0 \%$ \\
\hline & Muy alto & Recuento & 0 & 0 & 1 & 0 & 0 & 1 \\
\hline & & $\begin{array}{l}\text { \% dentro de } \\
\text { agotamiento emocional }\end{array}$ & $0,0 \%$ & $0,0 \%$ & $100,0 \%$ & $0,0 \%$ & $0,0 \%$ & $100,0 \%$ \\
\hline \multirow{2}{*}{\multicolumn{2}{|c|}{ Total }} & Recuento & 46 & 96 & 60 & 18 & 9 & 229 \\
\hline & & $\begin{array}{l}\% \text { dentro de } \\
\text { agotamiento emocional }\end{array}$ & $20,1 \%$ & $41,9 \%$ & $26,2 \%$ & $7,9 \%$ & $3,9 \%$ & $100,0 \%$ \\
\hline \multicolumn{9}{|c|}{ Pruebas de chi-cuadrado } \\
\hline & & \multicolumn{2}{|l|}{ Valor } & \multicolumn{2}{|l|}{ gl } & \multicolumn{3}{|c|}{ Sig. asintótica ( 2 caras) } \\
\hline \multicolumn{2}{|c|}{ Chi-cuadrado de Pearson } & $52,047 a$ & & 16 & & \multicolumn{3}{|c|}{, 000} \\
\hline \multicolumn{2}{|c|}{ Razón de verosimilitud } & 51,458 & \multicolumn{3}{|c|}{16} & \multicolumn{3}{|c|}{, 000} \\
\hline \multicolumn{2}{|c|}{ Asociación lineal por lineal } & 21,348 & \multicolumn{3}{|c|}{1} & \multicolumn{3}{|c|}{, 000} \\
\hline \multicolumn{2}{|c|}{$\mathrm{N}$ de casos válidos } & 229 & & & & & & \\
\hline
\end{tabular}

a. 15 casillas $(60,0 \%)$ han esperado un recuento menor que 5 . El recuento mínimo esperado es ,04

b. 15 casillas $(60,0 \%)$ han esperado un recuento menor que 5 . El recuento mínimo esperado es, 04 
Tabla 4. Tabla de contingencia entre despersonalización y logros personales para observar si se presenta el síndrome de burnout.

\begin{tabular}{|c|c|c|c|c|c|c|c|c|}
\hline & & & \multicolumn{5}{|c|}{ Logros personales } & \multirow[b]{2}{*}{ Total } \\
\hline & & & $\begin{array}{c}\text { No } \\
\text { presenta }\end{array}$ & Bajo & Regulares & Suficiente & Alto & \\
\hline \multirow[t]{10}{*}{ Despersonalización } & $\begin{array}{l}\text { No } \\
\text { presenta }\end{array}$ & Recuento & 24 & 16 & 5 & 2 & 0 & 47 \\
\hline & & $\begin{array}{l}\text { \% dentro de } \\
\text { Despersonalización }\end{array}$ & $51,1 \%$ & $34,0 \%$ & $10,6 \%$ & $4,3 \%$ & $0,0 \%$ & $100,0 \%$ \\
\hline & Bajo & Recuento & 18 & 48 & 22 & 3 & 0 & 91 \\
\hline & & $\begin{array}{l}\text { \% dentro de } \\
\text { Despersonalización }\end{array}$ & $19,8 \%$ & $52,7 \%$ & $24,2 \%$ & $3,3 \%$ & $0,0 \%$ & $100,0 \%$ \\
\hline & Regulares & Recuento & 3 & 27 & 18 & 7 & 7 & 62 \\
\hline & & $\begin{array}{l}\% \text { dentro de } \\
\text { Despersonalización }\end{array}$ & $4,8 \%$ & $43,5 \%$ & $29,0 \%$ & $11,3 \%$ & $11,3 \%$ & $100,0 \%$ \\
\hline & Suficiente & Recuento & 1 & 4 & 10 & 6 & 2 & 23 \\
\hline & & $\begin{array}{l}\text { \% dentro de } \\
\text { Despersonalización }\end{array}$ & $4,3 \%$ & $17,4 \%$ & $43,5 \%$ & $26,1 \%$ & $8,7 \%$ & $100,0 \%$ \\
\hline & Alto & Recuento & 0 & 1 & 5 & 0 & 0 & 6 \\
\hline & & $\begin{array}{l}\text { \% dentro de } \\
\text { Despersonalización }\end{array}$ & $0,0 \%$ & $16,7 \%$ & $83,3 \%$ & $0,0 \%$ & $0,0 \%$ & $100,0 \%$ \\
\hline \multirow[t]{2}{*}{ Total } & & Recuento & 46 & 96 & 60 & 18 & 9 & 229 \\
\hline & & $\begin{array}{l}\text { \% dentro de } \\
\text { Despersonalización }\end{array}$ & $20,1 \%$ & $41,9 \%$ & $26,2 \%$ & $7,9 \%$ & $3,9 \%$ & $100,0 \%$ \\
\hline
\end{tabular}

\section{Pruebas de chi-cuadrado}

\begin{tabular}{lccc} 
& Valor & gl & Sig. asintótica (2 caras) \\
\hline Chi-cuadrado de Pearson & $85,782 a$ & 16 &, 000 \\
Razón de verosimilitud & 83,780 & 16 &, 000 \\
Asociación lineal por lineal & 49,571 & 1 &, 000 \\
N de casos válidos & 229 & &
\end{tabular}

a. 13 casillas $(52.0 \%)$ han esperado un recuento menor que 5. El recuento mínimo esperado es 24 . 
En la tabla 5 se observa que no existe diferencias significativas del Burnout entre el personal docentes $y$ administrativo el valor $p=.584$ y se cumple que $p>\alpha(p>0.05)$.

Tabla 5. Diferencias significativas en la relación de la carga laboral y los rasgos del síndrome de burnout en el personal docente y administrativo.

\begin{tabular}{lllcc}
\hline & Tipo de labor que desempeña & N & $\begin{array}{c}\text { Rango } \\
\text { promedio }\end{array}$ & Suma de rangos \\
\hline $\begin{array}{l}\text { Rasgos del Síndrome de } \\
\text { Burnout }\end{array}$ & Personal docente & 105 & 112,11 & 11772,00 \\
& Personal administrativo & 123 & 116,54 & 14334,00 \\
& Total & $\mathbf{2 2 8}$ & & \\
Carga laboral & Personal docente & 105 & 113,96 & 11965,50 \\
& Personal administrativo & 123 & 114,96 & 14140,50 \\
& Total & $\mathbf{2 2 8}$ & & \\
\hline
\end{tabular}

Estadísticos de pruebaa

\begin{tabular}{lcc}
\hline & Rasgos del Síndrome de Burnout & Carga laboral \\
\hline U de Mann-Whitney & 6207,000 & 6400,500 \\
W de Wilcoxon & 11772,000 & 11965,500 \\
Z &,- 548 &,- 141 \\
Sig. asintótica (bilateral) &, 584 &, 888 \\
\hline
\end{tabular}

a. Variable de agrupación: Tipo de labor que desempeña.

Resultados en contraste son los hallados en los estudios de Vargas, M., Poveda., M., (2017) quien concluyo que hay una relación directa entre el Burnout y el desempeño laboral del personal administrativo, y que al no ser tratado genera características como cansancio emocional, despersonalización y falta de realización personal. Por medio de esta investigación, se concluye que se debe trabajar en torno a la preparación del personal administrativo sobre el Síndrome de Burnout, por tanto el investigador pone a consideración varias recomendaciones con el afán de colaborar no solo al personal administrativo, sino a la población de profesionales de similares características y usuarios en general de la Facultad de Ciencias Humanas y de la Educación de la Universidad Técnica de Ambato.
Aspectos que son corroborados por el análisis de contingencia en que la asociación de las variables burnout y carga laboral en los docentes y administrativos se presentan: 78,4\% (76 sujetos) mantienen una suficiente carga laboral presentando bajos rasgos de síndrome de burnout aunque también hay un grupo que no lo presentan $51,6 \%$ ( 49 sujetos) a diferencia de $79,4 \%$ ( 27 sujetos) que si presentan rasgos regulares del síndrome de burnout. Ante este comportamiento el Coeficiente Chi cuadrado con valor $=, 000$; lo cual indica que la asociación es muy significativa. Que existe asociación significativa entre la carga laboral y los rasgos de síndrome burnout del personal docente y administrativo de la Universidad Peruana Unión, Lima, 2017. 
Contrastes resultados presentan Marenco, Hernando(2016)quientambiénconcluyódimensiones de apoyo social asociadas con síndrome de burnout en docentes de media académica de la Corporación Universitaria Reformada, Barranquilla (Colombia). Los resultados que se encontró que el $23 \%$ de los docentes aquejó agotamiento emocional y el $22.5 \%$ despersonalización. Los niveles de apoyo social se mostraron con intensidad alta o media. Además, se observaron asociaciones significativas entre las dimensiones de apoyo y la despersonalización, mientras que la realización personal se asoció con formas emocionales, sociales e instrumentales de soporte. Concluyendo este autor en su estudio que se discute la implicación del apoyo social como un elemento relevante a considerar en la intervención preventiva del burnout en el profesorado.

Es importante resaltar que en el presente estudio la carga laboral en el personal de la UPeU presenta que el $67,7 \%$ tiene carga laboral suficiente a diferencia del $29,7 \%$ que presenta tener carga laboral baja y sólo un 2,7\% indica tener alta carga laboral, y en relación a los rasgos de posibilidad de presentar el Síndrome de Burnout el $41,5 \%$ no presenta ningún rasgo; el $42,4 \%$ presenta bajo rasgos de Burnout; el 14,8\% presenta rasgos medianamente; y sólo 1,3\% presenta rasgos moderadamente altos.

Datos que contrastan con González, Carrasquilla, Latorre, Torres, Villamil (2015) realizaron la investigación titulada "Síndrome de Burnout en docentes universitario". Donde sus resultados evidenciaron que el $66,66 \%$ de las docentes presentaron cansancio emocional bajo y el $26,66 \%$ cansancio emocional medio. 96,66\% nivel bajo de despersonalización y el $80 \%$ alto nivel de realización personal.

\section{CONCLUSIÓN}

El objetivo de la presente investigación fue determinar las diferencias significativas del burnout y la carga laboral en el personal docente y administrativo. Hallándose que no existen diferencias significativas del Burnout ni de la carga laboral entre el personal docente y administrativo de la Universidad Peruana Unión, Lima, 2017. Esto quiere decir que tanto docentes y administrativos están expuestos a los mismos factores de exposición a rasgos de burnout como de carga laboral.

\section{REFERENCIAS}

Barraza, J. (2013). Estrés Laboral y Burnout en docentes de Educación Superior en México". Departamento de Salud Pública de la Unidad Académica de Medicina (UAM), de la Universidad Autónoma de Nayarit (UAN). Recuperado: http://www.uan.edu.mx/es/ comunicados/estres-laboral-y-burnout-endocentes-de-educacion-superior-en-mexico

Carpio, G., y Requis, S. (2016). "Nivel de Síndrome de Burnout en enfermeras docentes de práctica que laboran en una Universidad Privada de Lima". http://revistaagora.com/index.php/ cieUMA/article/viewFile/60/58

Ceballos, V., y Paravic, T., Burgos, M., Barriga, O. (2014).Validación de escala subjetiva de carga mental de trabajo en funcionarios/as universitarios ciencia y enfermería xx (2): 73-82. http://www.scielo.cl/pdf/cienf/v20n2/art_08. pdf

González, G., Carrasquilla, D., Latorre, G., Torres, V., y Villamil, K. (2015). Síndrome de Burnout en docentes universitarios. Sede Santa Marta. Facultad de Enfermería de la Universidad Cooperativa de Colombia. VOL.31. Editorial Ciencias Médicas http://www. revenfermeria.sld.cu/index.php/enf/article/ view/902/138 3Aworkplace-stress-takes-atoll-on-individuals-employers-and-societies \&ltemid=135\&Lang=es 
Huamán, J., y Rodríguez, E. (2016). Síndrome de Burnout entre los trabajadores de la Municipalidad Alonzo de Alvarado Roque y la Municipalidad de Nueva Escuela de Psicologia, Upeu. Tarapoto. Recuperado de: http:// repositorio.upeu.edu.pe/bitstream/handle/ UPEU/717/Jesenia_Tesis_bachiller_ 2017. pdf?sequence $=1$

Instituto Nacional de Seguridad e Higiene en el Trabajo (2002) Carga mental de trabajo C/ Torrelaguna, 73 - 28027 Madrid ISBN: 84-7425605-4 Dep. Legal: M-12534-NIPO: 211-02-015-X Centro Nacional de Nuevas Tecnologías. Madrid Instituto Nacional de Seguridad e Higiene en el Trabajo

Ministerio de Salud (2006). Documento Técnico Normativo Plan nacional de salud mental Consejo nacional de salud. Recuperado de https://www.google.com.pe/search?q= ministerio+de+salud+mental+en+el+peru+ que+dice+sobre+el+burnout\&oq=ministerio++ s a l u d + mental+e n+e l + peru+que + dice+sobre+el+ burnout\&aqs=chrome. 69i57.252828j0 j7\&sourceid $=$ chromeqie $=$ UTF-8

Marenco, A., y Hernando, J. (2016). Dimensiones de apoyo social asociadas con síndrome de burnout en docentes de media académica. Corporación Universitaria Reformada, Barranquilla (Colombia). Recuperado: http:// www.redalyc.org/pdf/801/80146474001.pdf

Malander, N. (2016). Síndrome de Burnout y Satisfacción Laboral en Docentes de Nivel Secundario. Recuperado de: http://www.scielo. cl/pdf/cyt/v18n57/0718-2449-cyt-18-57-00177. pdf

Mansilla, F., (s/a) Síndrome de Burnout o síndrome de quemarse por el trabajo Cap. 4 Recuperado de: http://www.psicologia-online.com/ebooks/ riesgos/capitulo4_3.shtml

Maslach C, Jackson, S. Leiter, y M. Maslach Burnout Inventory. (1996). Manual. 3ed. Ed Palo Alto. California: Consulting Psychology Press. http:// www.mindgarden.com/products/mbi.htm
OPS, y OMS (2016). Estrés laboral es una carga para los individuos, los trabajadores y las sociedades. Washington, D.C., 28 de abril. Recuperado de http://www. paho.org/hq/index.php?option=com_ content\&view=articlectid $=11973 \%$

Riveri, R., Sánchez, A., Castellanos. M., Jay, B., y Ricardo, C. (2008). Síndrome de Burnout en el personal de enfermería del servicio de Terapia PolivalenteHospital.SaturninoLora.Recuperado de http://scielo.sld.cu/scielo.php?pid=S0864$03192008000200004 \&$ \&cript=sci_arttext

Rodríguez, A., Cruz, M., y., Merino, J. (2008). Validación de Burnout en Profesionales de Enfermeríaque trabajanencentrosasistenciales de la octava región. Chile. Recuperado de http://www.scielo.cl/scielo.php?pid=S071795532008000200010\&script=sci_arttext

Sánchez, C. (2011) “Estrés Laboral, Satisfacción en el Trabajo y Bienestar Psicológico en Trabajadores de una Industria Cerealera". Recuperado de: http://imgbiblio.vaneduc.edu. ar/fulltext/files/TC111836.pdf

Unión Nacional de Educadores (2005) Condiciones de Trabajo y Salud Docente Estudios de Casos en ARGENTINA, CHILE, ECUADOR, MEXICO, PERU Y URUGUAY. Recuperado de http://www. facso.uchile.cl/psicologia/epe/_documentos/ salud_docente/unesco_condiciones_trabajo_ salud_docente.pdf

Vargas, M., y Poveda., M. (2017). El síndrome de burnout en el desempeño laboral del personal administrativo de la Facultad de Ciencias Humanas y de la Educación de la Universidad Técnica de Ambato. De http://repositorio.uta. edu.ec/jspui/handle/123456789/25449 